\title{
Low Temperature Bonding of Metals by Deposition of Nanoparticles at the Interface
}

\author{
Toshio Takiya (Corresponding author) \& Naoaki Fukuda \\ Hitachi Zosen Corporation, Osaka 551-0022, Japan \\ Tel: 81-6-6551-9128Ｅ-mail: takiya@hitachizosen.co.jp
}

Ikurou Umezu \& Akira Sugimura

Department of Physics, Konan University, Kobe 658-8501, Japan

Tel: 81-78-435-2476 E-mail: umezu@konan-u.ac.jp

Shigeo Ueguri \& Hisao Yoshida

The Advanced Materials Processing Institute Kinki Japan, Hyogo 660-0083, Japan

Tel: 81-6-6412-7800Ｅ-mail: ueguri@ampi.or.jp

Min Han

Department of Materials Science and Engineering Nanjing University

22 Hankou Road, Nanjing 210093, China

Tel: 86-25-8368-6248_E-mail: sjhanmin@nju.edu.cn

Received: August 4, 2011 Accepted: August 26, $2011 \quad$ Published: February 1, 2012

doi:10.5539/apr.v4n1p42 URL: http://dx.doi.org/10.5539/apr.v4n1p42

The research is financed by the Hyogo Prefecture COE Program and Amagasaki City Research Grant in Japan.

\begin{abstract}
Copper nanopartices having some tens nanometer in diameter were prepared by pulsed laser ablation in a helium background gas to form active interface of copper-to-copper bonding. The nanoparticles deposited on $\mathrm{Cu}$ plate were annealed in hydrogen gas atmosphere. The sintering starts from below $473 \mathrm{~K}$ and boundary of nanoparticles was hardly observed above $673 \mathrm{~K}$. Bonding of copper-to-copper metal pieces has been carried out by deposition of copper nanoparticles on the test piece as active interface followed by annealing at $673 \mathrm{~K}$. The measured shear strength of the copper-to-copper bonding was $106 \mathrm{MPa}$, which is much higher value than that when solder was used as bonding material. The sintering process of $\mathrm{Cu}$ nanoparticles is discussed.
\end{abstract}

Keywords: Metal nanoparticle, Laser ablation, Low temperature bonding

\section{Introduction}

Bonding of metal is important to construct fine mechanical parts, heat sinks and so on. Thermal process is necessary for conventional bonding methods such as diffusion bonding, melted alloy bonding, hot iso-static pressing and silver brazing. Thermal stress is induced in the interface due to the difference in thermal expansion between the bonded parts, and the stress causes warp of the bonded materials. Therefore, low temperature bonding is desired to reduce these problems. One of the methods to accomplish low temperature bonding is to use nanoparticle paste as a bonding material, since the melting point of nanoparticle decreases with decreasing the size. However, strength of bonding by using nanoparticle paste is relatively low compared with conventional methods.

Since the bonding is accomplished by sintering of nanoparticle, it is important to reveal the sintering phenomena 
of the metallic nanoparticles to improve the strength of bonding. Effects of super-plasticity and diffusivity on the mechanical strength of nano-structured alloy layer prepared using an electron beam deposition method were studied (Mukai, et al., 2003; Somekawa, et al., 2003, Somekawa, et al., 2004), and interaction of a metallic nanoparticle with adjacent one during electron beam irradiation was also investigated from the viewpoint of nanoparticle bonding (Xu \& Tanaka, 1999). Pulsed laser ablation (PLA) is well known as one of the good physical vapor deposition (PVD) methods to prepare high purity metal nanoparticles, so that it is a promising technique to accomplish low temperature bonding. Effects of metallic nanoparticles prepared by PLA on the bonding under the low temperature were investigated in the present paper.

\section{Experiment}

Copper nanoparticles were deposited by PLA of $\mathrm{Cu}$ target in helium background gas. A YAG laser (wavelength: $355 \mathrm{~nm}$, energy: $11 \mathrm{~mJ} /$ pulse) was focused on the $\mathrm{Cu}$ target to a beam spot radius of $0.5 \mathrm{~mm}$. The target-substrate distance and helium gas pressure were varied from 10 to $40 \mathrm{~mm}$ and from 80 to $1333 \mathrm{~Pa}$, respectively.

Mean diameter of nanoparticles was measured by atomic force microscope (AFM). The diameters of the nanoparticles ranged from $10 \mathrm{~nm}$ to several tens of $\mathrm{nm}$. The aggregation of the nanoparticles is the smallest when the helium background gas pressure and substrate-target distance were $80 \mathrm{~Pa}$ and $40 \mathrm{~mm}$ in our experimental condition. We used this condition to form nanoparticles as a bonding material.

The deposited sample was annealed up to $673 \mathrm{~K}$ in an annealing chamber. The base pressure of annealing chamber was $0.01 \mathrm{~Pa}$ and the hydrogen gas was introduced in the chamber. Temperature was elevated to desired value in $10 \mathrm{~min}$ and kept at constant value for $5 \mathrm{~min}$.

The test pieces to measure shear strength of the bonding are composed of cylinder and plate as shown in Fig. 1(a). The surface roughness of the test pieces was less than $10 \mathrm{~nm}$ of Ra and the flatness was less than $0.3 \mu \mathrm{m}$. Nanoparticle layer of $1.0 \mu \mathrm{m}$ in thickness was deposited on the plate piece. After cylindrical pieces was placed on the plate pieces as shown in Fig. 1(a), a pressure of $13 \mathrm{MPa}$ was applied at the interface and hydrogen gas was introduced in the annealing chamber at rate of $2 \mathrm{l} / \mathrm{min}$ during annealing, in order to enhance the surface activity of nanoparticles and bond strength. A schematic view of the set up used to evaluate the shear strength of the bonded test piece is shown in Fig. 1(b). The strength of bonding is determined with the applied force when it is ruptured at the interface.

\section{Results and Discussions}

\subsection{Low temperature bonding}

It is well known that the melting point decrease by decreasing the size of materials (Qi, et al., 2003). The decrement of the melting point $\Delta T$ for a nanoparticle of diameter $d$ is expressed as follows (Ragone, 1995):

$$
\Delta T=-\frac{4 V_{s} \gamma_{l-s} T_{m}}{\Delta H_{m}} \frac{1}{d}
$$

where, $V_{s}$ is the volume per mole, $\Delta H_{m}$ the melting enthalpy per mole, $\gamma_{l-s}$ the interface tension between the liquid and solid phase and $T_{m}$ the melting point for the bulk. If we assume that the material is copper and $\gamma_{l-s}$ is half the value of bulk's surface tension, $\Delta T$ is about $160 \mathrm{~K}$ for a copper nanoparticle having a diameter of $10 \mathrm{~nm}$.

The decrease in the melting point results in the decrease in the sintering temperature and realizes diffusion bonding at relatively low temperature.

\subsection{Sintering phenomena of nanoparticles}

Figure 2(a) shows AFM image of nanoparticles before annealing, and Figs. 2(b), (c) and (d) show those after annealing at $473 \mathrm{~K}, 573 \mathrm{~K}$ and $673 \mathrm{~K}$, respectively. As can be seen from the images, size of nanoparticles increased with annealing temperature.

According to sintering process theory, the final diameter of a nanoparticle, $d_{f}$, depends on the annealing temperature (Koch \& Friedlander, 1990). Particle growth rate can be expressed by surface area of a nanoparticle

$$
\frac{d a}{d t}=-\frac{1}{\tau}\left(a-a_{f}\right)
$$

Where, $t$ is the time, $\tau$ is the characteristic time of particle growth by sintering, $a$ is the surface area and $a_{f}$ the value of the surface area at a final size. The particle growth rate depends on characteristic time which is determined by the two main types of the diffusion i.e. the lattice diffusion and the grain boundary diffusion. 
Characteristic time of the lattice diffusion, $\tau_{l}$, is proportionate to the third power of the particle diameter, $d^{3}$, and temperature, $T$, and it is inversely proportional to the surface energy, $\gamma$, the diffusion constant for lattice diffusion, $D_{l}$ and the atomic volume, $\Omega$. The practical expression regarding $\tau_{l}$ is inferred from information addressed in some papers (Greer \& Street, 2007; Arcidiacono et al., 2004; Maximenko \& Olevsky, 2004). Here, the following expression was adopted.

$$
\tau_{l}=\frac{k T d^{3}}{\gamma D_{l} \Omega}=\frac{k T d^{3}}{\gamma D_{l 0} \Omega} \exp \left(\frac{\varepsilon}{k T}\right)
$$

Where, $k$ is the Boltzman constant, $D_{l 0}$ the vibration constant for lattice diffusion, $\varepsilon$ the activity energy for diffusion.

As shown in Eq. (3), the characteristic time $\tau_{l}$ for lattice diffusion vary against temperature. While the role of grain boundaries for enhancing atomic diffusion is important (Zhu \& Averback, 1995), the characteristic time $\tau_{b}$ for grain boundary diffusion is always shorter than the time $\tau_{l}$ for lattice diffusion under the low temperature conditions. The ratio of the characteristic times, $\alpha$, is as following (Ragone, 1995):

$$
\alpha=\frac{\tau_{l}}{\tau_{b}}=\left(\frac{2 \delta D_{b}}{D_{l}}\right) \frac{1}{d}
$$

Here, $\delta$ is the width of grain boundary and $D_{b}$ is the diffusion coefficient for grain boundary diffusion. If the structure of nanoparticle is face-centered cubic, the degree of the grain boundary effect, $\alpha$, is calculated as follows (Ragone, 1995).

$$
\alpha=\frac{6.0 \times 10^{8}}{d} \times \exp \left(8.1 \times \frac{T_{m}}{T}\right)
$$

Here, $T_{m}$ is the melting point of the metal.

Since the characteristic time for grain boundary diffusion, $\tau_{b}$, is proportional to the fourth power of particle diameter according to Eq.(4), the smaller the particle diameter the larger the effect of grain boundary diffusion. If the effect of grain boundary diffusion is taken in account to the calculation of the particle growth rate by Eq. (2), supposing $\tau=\tau_{b}=\tau_{l} / \alpha$, the characteristic time $\tau$ can be estimated as $6.81 \times 10^{2} \mathrm{~s}, 2.67 \mathrm{~s}$ and $5.58 \times 10^{-2} \mathrm{~s}$ at temperatures of $473 \mathrm{~K}, 573 \mathrm{~K}$ and $673 \mathrm{~K}$, respectively. The parameters used for the above estimation are shown in Table 1.

The final diameter $d_{f}\left(=\sqrt{a_{f} / \pi}\right)$ can be estimated from the value of $\tau$ in Eq. (2). By solving the equation for the surface area $a_{f}$, we obtained the following equation:

$$
a_{f}=\frac{a_{1}-a_{0} \exp \left(-\frac{t_{1}}{\tau}\right)}{1-\exp \left(-\frac{t_{1}}{\tau}\right)}
$$

Here, the initial surface area, $a_{0}$, or the initial diameter, $d_{0}$, was estimated from AFM images of as-deposited sample while the surface area $a_{l}$ or the diameter $d_{l}$ was estimated from that of annealed sample for annealing time $t_{l}=5 \mathrm{~min}$.

Figure 3 illustrates the changes in particle diameter as a function of time, which were calculated from Eq. (2) using specific values for temperature. Comparing particle growth at different temperature conditions, we found that the particle growth starts earlier, the particle growth rate is faster, and the final size is larger at relatively higher temperature.

Figure 4 shows mean diameter of particles measured from AFM images in Fig.2. Solid line is the regression line by the method of least square from the final size of particles calculated by eq. (6). As shown in the figure, the final particle size increases exponentially as the temperature increases. The mean diameter of nanoparticles prepared at $473 \mathrm{~K}$ is slightly lower than the solid line because the annealing time of $5 \mathrm{~min}$ is insufficient for annealing temperature of $473 \mathrm{~K}$.

It is necessary to reduce the value of $\tau$, in order to enhance the sintering process. It can be deduced from Eq. (3) that it is effective to not only increase temperature but also to decrease the initial diameter of nanoparticle. In the 
viewpoint of low temperature bonding, however, it is preferable to keep temperature as low as possible by decreasing the size of nanoparticle before annealing.

\subsection{Shear strength test}

The bonding of cylinder and plate was performed at $673 \mathrm{~K}$. The results of shear strength values are shown in Table 2. Here, the notation copper/nanoparticle/copper indicates the result of the present bonding method, while the notations "copper/none/copper" and "copper/solder/copper" indicate conventional diffusion bonding without any adhesive material and bonding by Sn-Ag soldering, respectively. Although the shear strength was about from 30 to $40 \mathrm{MPa}$ in the case of the diffusion bonding and soldering, the shear strength of over $66 \mathrm{MPa}$ was obtained in the case of bonding with nanoparticles. This is considered to be the effects of sintering at $673 \mathrm{~K}$ discussed earlier. In a certain instance, the shear strength of $106 \mathrm{MPa}$ was achieved as a maximum value. This means that the existence of nanoparticles is effective to the bonding of materials.

\section{Conclusions}

In conclusion, copper nanoparticles were formed by PLA of copper target in helium background gas. We investigated availability of the nanoparticles as an interface layer between copper-to-copper for low temperature bonding. After annealing, nanoparticles adhered to each other at temperature of $673 \mathrm{~K}$. The shear strength of the sample prepared by the present method was more than twice larger than that of solder bonding. Copper-to-copper bonding using copper nanoparticles is effective to strengthen the bonding of mechanical parts at relatively low temperature.

\section{Acknowledgements}

This research was undertaken, in part, thanks to funding from the Hyogo Prefecture COE Program and Amagasaki City Research Grant in Japan. Authors are also thankful to Hiroshi Kaita and Takaaki Tonoura in Neis corporation for their valuable discussions.

\section{References}

Arcidiacono, S., Bieri, N. R., Poulikakos, D., \& C. P. Grigoropoulos. (2004). On the coalescence of gold nanoparticles. Int. J. Multiphase Flow, 30, 979-994. http://dx.doi.org/10.1016/j.ijmultiphaseflow.2004.03.006

Greer, J. R. \& Street, R. A. (2007). Thermal cure effects on electrical performance of nanoparticle silver inks. Acta Mater., 55, 6345-6349. http://dx.doi.org/10.1016/j.actamat.2007.07.040

Koch, W. \& Friedlander, S. K. (1990). The effect of particle coalescence on the surface area of a coagulating aerosol. Journal of Colloid and Interface Science, 140-2, 419-427. http://dx.doi.org/10.1016/0021-9797(90)90362-R

Maximenko, A. L. \& Olevsky, E. A. (2004). Effective diffusion coefficients in solid-state sintering. Acta Mater., 52, 2953-2963. http://dx.doi.org/10.1016/j.actamat.2004.02.042

Mukai, T., Suresh, S., Kita, K., Sakai, H., Kobayashi, N., Higashi, K., \& Inoue, A. (2003). Nanostructured Al-Fe alloys produced by e-beam deposition: static and dynamic tensile properties. Acta Mater., 51, 4197-4208. http://dx.doi.org/10.1016/S1359-6454(03)00237-4

Qi, W. H., Wang, M. P., \& Xu, G. Y. (2003). The particle size dependence of cohesive energy of metallic nanoparticles. Chem. Phys. Lett., 372, 632-634. http://dx.doi.org/10.1016/S0009-2614(03)00470-6

Ragone, D. V. (1995). Thermodynamics of materials, Volume II, John Wiley \& Sons, Inc., New York.

Somekawa, H., Watanabe, H., Mukai, T., \& Higashi, K. (2003). Low temperature diffusion bonding in a $\begin{array}{lllll}\text { superplastic AZ31 magnesium alloy. Scripta Mater., } & 48, & 1249-1254 .\end{array}$ http://dx.doi.org/10.1016/S1359-6462(03)00054-X

Somekawa, H., Tanaka, T., Sasaki, H., Kita, K., Inoue, A., \& Higashi, K. (2004). Diffusion bonding in ultra fine-grained Al-Fe alloy indicating high-strain-rate superplasticity. Acta Mater., 52, 1051-1059. http://dx.doi.org/10.1016/j.actamat.2003.10.036

Xu, B. S., \& Tanaka, S. (1999). Behavior and bonding mechanisms of aluminum nanoparticles by electron beam irradiation. Nanostructured Materials, 12, 915-918. http://dx.doi.org/10.1016/S0965-9773(99)00266-4

Zhu, H. \& Averback, R. S. (1995). Materials Science \& Engineering, A204, 96-100. 
Table 1. Parameters used for the copper calculation

\begin{tabular}{ccc}
\hline Physical Quantity & $\begin{array}{c}\text { Symbols and } \\
\text { Units }\end{array}$ & Values \\
\hline Vibration Factor & $D_{l 0}\left[\mathrm{~m}^{2} / \mathrm{s}\right]$ & $3.54 \times 10^{-4}$ \\
Surface Energy & $\gamma\left[\mathrm{J} / \mathrm{m}^{2}\right]$ & 1.60 \\
Activation Energy & $\varepsilon[\mathrm{J}]$ & $3.66 \times 10^{-19}$ \\
Atomic Volume & $\Omega\left[\mathrm{m}^{3}\right]$ & $1.18 \times 10^{-29}$ \\
Melting Temperature & $T_{m}[\mathrm{~K}]$ & 1356 \\
\hline
\end{tabular}

Table 2. Experimental results of shear strength

\begin{tabular}{ccc}
\hline Test piece & $\begin{array}{c}\text { Shear force } \\
(\mathrm{N})\end{array}$ & $\begin{array}{c}\text { Shear strength } \\
(\mathrm{MPa})\end{array}$ \\
\hline copper/nanoparticle/copper & $>1251$ & $>66$ \\
copper/none/copper & 735 & 38 \\
copper/solder/copper & 710 & 36 \\
\hline
\end{tabular}

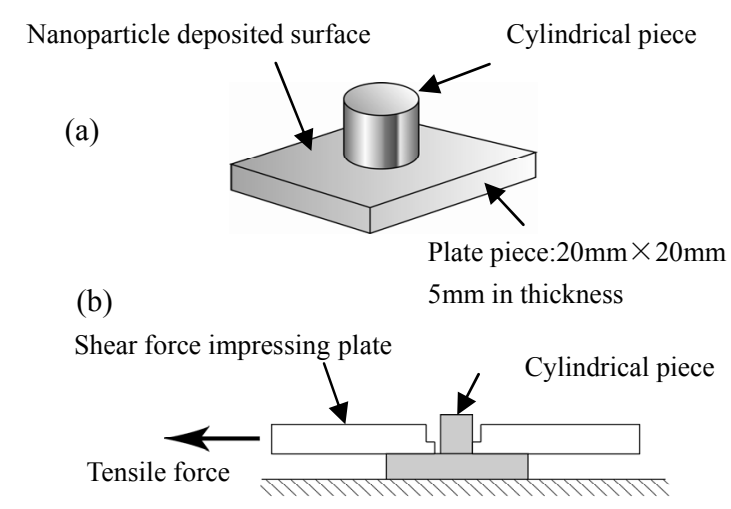

Figure 1. (a) Shape of test piece to measure shear strength; (b) A schematic view of set up to measure shear strength 


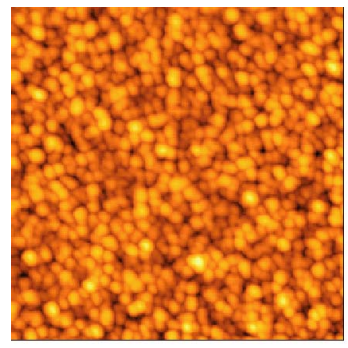

(a) before annealing

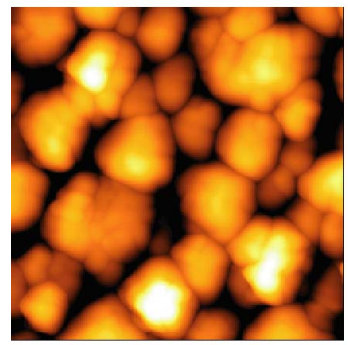

(c) after annealing at $573 \mathrm{~K}$

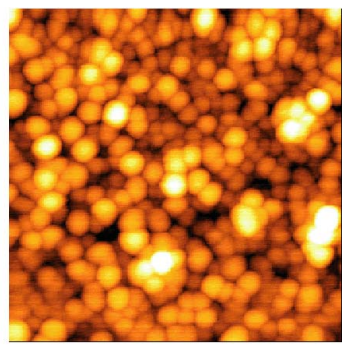

(b) after annealing at $473 \mathrm{~K}$

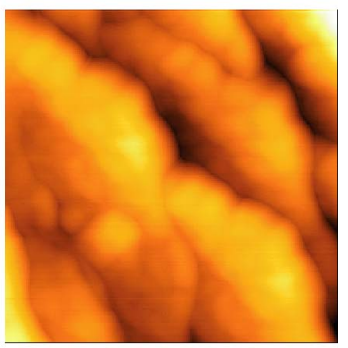

(d) after annealing at $673 \mathrm{~K}$

Figure 2. Effect of annealing on the sintering of copper nanoparticles measured by AFM (field of view: $500 \times 500 \mathrm{~nm}$ )

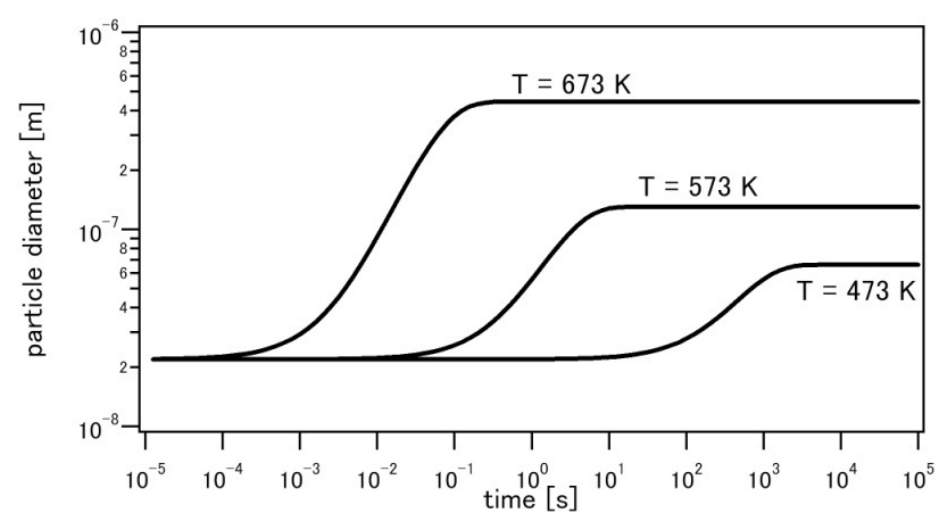

Figure 3. Estimated particle size as a function of annealing time at different annealing temperature

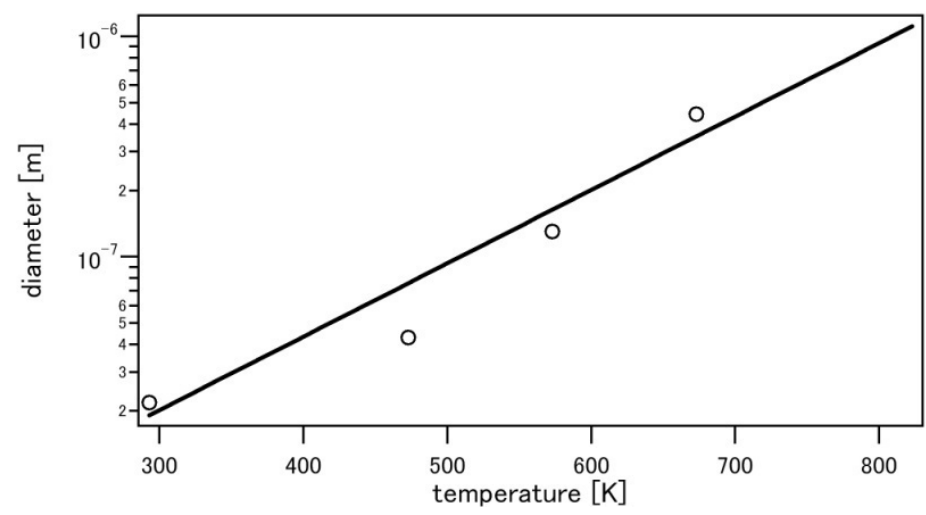

Figure 4. Open circles show particle diameter as a function of annealing temperature estimated from AFM images. Straight line is a result of least mean fitting 